\title{
artigo
}

Campos, D.P. Kanaan, S.; Lourenção, L.G.; Lopes, V.G.S.; Xavier, A.R.;

Perfil clínico e epidemiológico de gestantes com HIV positivo atendidas em um hospital municipal de Niterói

\section{Perfil clínico e epidemiológico de gestantes com HIV positivo atendidas em um hospital municipal de Niterói}

\author{
Clinical and epidemiological profile of pregnant HIV positive pregnants at a municipal hospital in Niterói \\ Perfil clínico y epidemiológico de embarazadas positivas por VIH embarazadas en un hospital municipal de Niterói
}

\begin{abstract}
RESUMO
Objetivo: Avaliar o perfil epidemiológico das gestantes HIV positivo, acompanhadas no Ambulatório do Hospital Municipal Carlos Tortelly de Niterói - RJ. Metodologia: Trata-se de um estudo epidemiológico retrospectivo, quantitativo e descritivo. Consta de 103 gestantes HIV positivo na faixa etária 18 e 45 anos, atendidas no ambulatório de SIDA/Hospital Municipal Carlos Tortelly (HMCT), durante o período de dezembro de 2009 a dezembro de 2015. Resultados: A faixa etária predominante entre 21 a 30 anos (57,3\%), sendo que 40,8\% tinham ensino fundamental, 30,1\% com renda familiar em torno de um salário mínimo, e a maioria 60,2\% procedia no Município de Niterói. A detecção do HIV, majoritariamente, ocorreu antes da gravidez $(57,3 \%$ ), e apenas $14,6 \%$ das gestantes não consumia alguma substância química durante a gestação e 45,6\% não sofreram infecção oportunista durante o acompanhamento, 28,2\% já tinham dois ou mais filhos. Cesária não eletiva foi a maior forma de escolha para o nascimento $(53,4 \%$ ). Conclusão: $O$ perfil se caracteriza por situação socioeconômica vulnerável, com baixo nivel de escolaridade, e o diagnóstico anterior precedem a gestação atual. A realização do pré-natal, e a terapia TARV na gestação e no parto foi alto. Para tanto, faz-se necessário o melhoramento das informações e ações direcionadas à ampliação da atenção às mulheres, cuja garantia integral e o diagnóstico precoce do HIV constituem artifícios importantes na redução da transmissão vertical.
\end{abstract}

DESCRITORES: Promoção da Saúde; Universidade; Enfermagem; Serviços de Saúde para Estudantes.

\section{ABSTRACT}

Objective: To evaluate the epidemiological profile of HIV positive pregnant women, followed up at the Ambulatory of Hospital Municipal Carlos Tortelly de Niterói - RJ. Methodology: This is a retrospective, quantitative and descriptive epidemiological study. There are 103 HIV positive pregnant women aged between 18 and 45 years, attended at the AIDS outpatient clinic / Hospital Municipal Carlos Tortelly (HMCT), from December 2009 to December 2015. Results: The predominant age group between 21 to 30 years (57.3\%), with $40.8 \%$ having elementary education, $30.1 \%$ with family income around one minimum wage, and the majority $60.2 \%$ came from the city of Niterói. The detection of HIV, mostly, occurred before pregnancy $(57.3 \%)$, and only $14.6 \%$ of pregnant women did not consume any chemical substance during pregnancy and $45.6 \%$ did not suffer opportunistic infection during the follow-up, $28.2 \%$ already had two or more children. Non-elective cesarean was the greatest form of choice for birth (53.4\%). Conclusion: The profile is characterized by a vulnerable socioeconomic situation, with a low level of education, and the previous diagnosis precedes the current pregnancy. Prenatal care and ART therapy during pregnancy and childbirth were high. To this end, it is necessary to improve information and actions aimed at expanding care for women, whose integral guarantee and the early diagnosis of HIV are important devices in the reduction of vertical transmission. DESCRIPTORS: Health Promotion; University; Nursing; Student Health Services.

\section{RESUMEN}

Objetivo: evaluar el perfil epidemiológico de las embarazadas VIH positivas, seguidas en el ambulatorio del Hospital Municipal Carlos Tortelly de Niterói - RJ. Metodología: se trata de un estudio epidemiológico retrospectivo, cuantitativo y descriptivo. Hay 103 mujeres embarazadas VIH positivas de entre 18 y 45 años, atendidas en la clínica ambulatoria de SIDA/Hospital Municipal Carlos Tortelly (HMCT), desde diciembre de 2009 hasta diciembre de 2015. Resultados: el grupo de edad predominante entre 21 y 30 años $(57.3 \%)$, con $40.8 \%$ con educación primaria, $30.1 \%$ con ingresos familiares alrededor de un salario mínimo, y la mayoría $60.2 \%$ provino de la ciudad de Niterói. La detección del VIH, principalmente, ocurrió antes del embarazo (57.3\%), y solo el $14.6 \%$ de las mujeres embarazadas no consumieron ninguna sustancia química durante el embarazo y el $45.6 \%$ no sufrió una infección oportunista durante el seguimiento, 28.2 \% ya tenía dos o más hijos. La cesárea no electiva fue la mejor forma de elección para el nacimiento (53,4\%). Conclusión: el perfil se caracteriza por una situación socioeconómica vulnerable, con un bajo nivel educativo, y el diagnóstico previo precede al embarazo actual. La atención prenatal y la terapia ART durante el embarazo y el parto fueron altas. Para ello, es necesario mejorar la información y las acciones destinadas a ampliar la atención a las mujeres, cuya garantía integral y el diagnóstico precoz del VIH son dispositivos importantes en la reducción de la transmisión vertical.

DESCRIPTORES: Promoción de la Salud; Universidad; Enfermería; Servicios de Salud Estudiantil.

RECEBIDO EM: 13/02/2020 APROVADO EM: 14/02/2020 


\section{Danielle Pinheiro Campos}

Farmacêutica. Mestre Em Saúde Materno-Infantil, Universidade Federal Fluminense. Industria Farmacêutica Multinacional. https://orcid.org/0000-0003-1642-6359

\section{Salim Kanaan}

Médico. Mestre em Biofísica. Professor Adjunto do Departamento de Patologia da Faculdade de Medicina da Universidade Federal Fluminense. https://orcid.org/0000-0001-5759-731X

\section{Luciano Garcia Lourenção}

Enfermeiro. Doutor em Ciências da Saúde. Professor Titular-Livre da Escola de Enfermagem da Universidade Federal do Rio Grande. https://orcid.org/0000-0002-1240-4702

\section{Vânia Glória Silami Lopes}

Médica. Doutora em Anatomia Patológica e Patologia Clínica. Professora Titular do Departamento de Patologia da Faculdade de Medicina da Universidade Federal Fluminense. https://orcid.org/0000-0002-6656-5763

\section{Analúcia Rampazzo Xavier}

Farmacêutica-Bioquímica. Doutora em Bioquímica. Professora Associada do Departamento de Patologia da Faculdade de Medicina da Universidade Federal Fluminense. Chefe do Departamento de Patologia da Faculdade de Medicina da Universidade Federal Fluminense. https://orcid.org/0000-0003-4501-1857

\section{INTRODUÇÃO}

A Síndrome da Imunodeficiência Adquirida (AIDS) foi primeiramente descrita no início da década de 80, mais precisamente em 1981 nos Estados Unidos, porém observa-se que a quantidade de portadores tem aumentado significativamente nos últimos anos ${ }^{(1,2)}$. É ocasionada pela presença e multiplicação do vírus da imunodeficiência humana (HIV), que com o passar dos anos, leva a um quadro clínico que, lentamente, compromete o sistema imunológico da pessoa, culminando na $\operatorname{AIDS}^{(3,4)}$.

A AIDS e as infecções oportunistas provocadas pela deficiência imunológica são sempre o foco de atenção médica em todos os países do mundo. O fato de ser, até o presente momento, uma doença incurável e mortal, faz da AIDS um objeto importante para o estudo, tanto para as ciências médicas como para as ciências sociais e comportamentais. Apesar dos esforços para encontrar a cura da doença, o ramo das ciências médicas obteve um avanço considerável em relação ao tratamento, assim como, foram aprofundados os conhecimentos sobre os mecanismos de ação do vírus no organismo humano ${ }^{(4)}$.

Esta síndrome é uma das situações clínicas que mais preocupam a população mundial nos dias atuais, devido, principal- mente, ao seu caráter endêmico e pelo fato de não diferenciar sexo, idade, condições socioeconômicas, além de ser altamente letal. Concomitante, a este fato, atitudes, comportamentos de risco e intercorrências clínicas comumente associadas à presença do HIV, são alguns fatores complicadores difíceis de controlar ${ }^{(5)}$. A transmissão do HIV pode ocorrer por: via sanguínea, relação sexual desprotegida ou transmissão vertical (da mãe para o feto). Nesta, o contágio pode ocorrer durante o período gestacional, no momento do parto ou através da amamentação ${ }^{(6)}$.

Segundo o Programa das Nações Unidas para o combate à AIDS (UNAIDS), há no mundo 36,7 milhões de pessoas infectadas pelo vírus HIV, sendo que cerca de $40 \%$ são mulheres. O número de mulheres infectadas pelo HIV vem crescendo, e a humanidade encontra um cenário de feminização do HIV/AIDS. No mundo, cerca de 17,8 milhões de mulheres são HIV positivo, com idade superior à 15 anos $^{(5)}$.

Esse número de mulheres infectadas resulta em número também crescente de crianças nascendo com o vírus HIV. Desde a década de 80 , a transmissão vertical (TV), ou seja, a transmissão do HIV da mãe infectada para o filho por via transplacentária, no trabalho de parto, parto ou amamentação, tem predominado de for- ma marcante, sendo o primeiro caso notificado e confirmado de soropositividade por este tipo de exposição em $1985^{(7)}$.

Ao longo dos trinta anos da história da AIDS no Brasil, a epidemia apresentou alterações nas características epidemiológicas. A partir da década de 1990, ocorreu o aumento no número de casos da doença entre usuários de drogas injetáveis, seguido do aumento de casos em homens heterossexuais e mulheres ${ }^{(8)}$. Observou-se a interiorização, pauperização e feminização da epidemia, evidenciando a disseminação da infecção por HIV para municípios menores e com renda per capita mais baixa ${ }^{(9)}$, aumento do número de casos em pessoas com baixa escolaridade e maior vulnerabilidade das mulheres à infecção pelo vírus ${ }^{(10)}$.

Considerando a importância dos agravos ocasionados pela doença e da complexidade do tratamento, a cobertura a qualidade do pré-natal são fatores significativos para que as medidas a serem tomadas contribuam consideravelmente para minimização da mortalidade materna e frequência da TV do HIV. Assim, o objetivo deste trabalho é encontrar maior taxa de sucesso na prevenção da TV nas gestantes HIV positivo que fizerem o uso correto da medicação antirretroviral (ARV) e pré-natal completo. Ao mesmo tempo, esperamos achar uma correlação positiva entre maior 
precocidade no início do tratamento com o desfecho positivo na prevenção da transmissão do HIV para os recém-natos e, com isso, fornecer subsídios para programas multiprofissionais de prevenção.

A importância dos estudos epidemiológicos sobre as características populacionais dos indivíduos infectados com o HIV e AIDS para os programas de prevenção, associada às poucas informações epidemiológicas em Niterói, RJ, o presente trabalho buscou conhecer o perfil das gestantes HIV-positivo que são acompanhadas em Hospital Municipal de Niterói - RJ; atualmente considerado polo de tratamento de HIV pelo Ministério da Saúde, através do Programa IST/AIDS.

Objetivou-se avaliar o perfil epidemiológico das gestantes HIV positivo acompanhadas no Ambulatório do Hospital Municipal da cidade de Niterói - RJ.

\section{METODOLOGIA}

Trata-se de um estudo epidemiológico retrospectivo, descritivo e qualitativo.

O estudo foi realizado em Hospital da rede municipal da cidade de Niterói, Rio de Janeiro. em Niterói, Rio de Janeiro. Esta unidade ambulatorial possui sete consultórios adultos e dois infantis. Atende pacientes vítimas de estupros ou que foram contaminadas por parceiros dependentes de drogas e/ou com Infecções Sexualmente Transmissíveis (ISTs), e pacientes com agravos decorrentes de suas condições clínicas. O Hospital Municipal Carlos Tortelly é considerado um centro de referência do Município de Niterói em IST e HIV/AIDS.

A população do estudo compreendeu as gestantes HIV positivo, entre 18 e 45 anos, que estiveram em acompanhamento no ambulatório de SIDA/HMCT, durante o período de dezembro de 2009 a dezembro de 2015.

Os critérios de inclusão no estudo foram mulheres grávidas HIV positivo com faixa etária 18 a 45 anos, independentemente do desfecho, isto é, do sucesso ou insucesso da TV. Foram excluídas as gestantes HIV positivo cujos dados não estavam devidamente preenchidos nos prontuários.

A partir de levantamento bibliográfico nacional e internacional acerca do HIV em gestantes, foi desenvolvido um instrumento para coleta de dados, do tipo formulário semiestruturado, sendo que as variáveis foram obtidas a partir da análise documental dos registros de consultas, realizadas pela equipe multiprofissional do serviço, sendo os dados compilados para um instrumento elaborado pelos autores.

Foram avaliadas as seguintes variáveis das gestantes:

a) Variáveis socioeconômicas e demográficas:

* Renda familiar: variável numérica estratificada em: $\mathrm{R} \$ 100,00$ a $\mathrm{R} \$ 600,00$, $\mathrm{R} \$ 700,00$ a $\mathrm{R} \$ 1.200,00, \mathrm{R} \$ 1.300,00 \mathrm{a}$ $\mathrm{R} \$ 1.800,00, \mathrm{R} \$ 1.900,00$ a $\mathrm{R} \$ 2.200,00$, $\mathrm{R} \$ 2.300,00 \mathrm{a} \mathrm{R} \$ 2.800,00 \mathrm{e} \mathrm{R} \$ 2.900,00$ a $\mathrm{R} \$ 3.500,00$.

* Faixa etária: variável numérica estratificada em décadas $(10,20,30,40$ ou mais).

* Escolaridade: variável categórica ordinal estratificada em: Analfabeta, Ensino fundamental, Ensino Médio e Nível Superior.

b) Perfil clínico das gestantes:

* Data de atendimento: variável numérica estabelecida em anos completos.

* Procedência: variável nominal.

* Números de gestações: variável numérica. * Momento do diagnóstico do HIV em relação ao pré-natal: variável nominal categorizada em antes, durante, no parto, sem informação.

* Utilização de drogas injetáveis: variável nominal categorizada em sim, não e sem informações.

* Abortos anteriores: variável numérica.

* Outras IST: variável nominal categorizada em nenhuma, Sífilis, Herpes e sem informação.

* Uso do antibiótico: variável nominal categorizada em sim, não ou dado não encontrado.

*Tipo de parto: variável nominal categorizada em cesárea eletiva, cesárea não eletiva e parto normal.
A amostra do estudo foi composta por 103 prontuários cadastrados no ambulatório de SIDA/HMCT. Os procedimentos referentes à coleta de dados iniciaram imediatamente após a aprovação do Comitê de Ética. A coleta de dados ocorreu no período de março de 2017 a 2018 . O estudo foi aprovado pelo Comitê de Ética em Pesquisa com Seres Humanos da Universidade de Medicina da Universidade Federal Fluminense, em 20 de março de 2017, CAAE n ${ }^{\circ}$ 56261816.8.0000.5243, sob Parecer n..$^{\circ} 1.972 .883$, seguindo as recomendações constantes na Resolução CNS n. ${ }^{\circ} 466 / 2012$.

Os dados obtidos foram digitados em planilha do Microsoft Excel $^{\circ}$ e submetidos à análise descritiva, utilizando-se o programa Statistical Package for the Social Sciences (SPSS), versão 18.0. Na análise estatística foi realizada distribuição de frequência absoluta e relativa para a construção do perfil das gestantes. Os resultados foram apresentados por meio de tabelas.

\section{RESULTADOS}

Foram avaliadas 103 gestantes HIV positivo, pode retirar a identificação pois já está descrito nos métodos, no período de 2009 a 2015. Houve maior número de atendimentos nos anos de 2009 (27,2\%) e 2015 (20,4\%). As pacientes eram procedentes, prevalentemente, de Niterói $(60,2 \%)$, com idade entre 21 a 30 anos (57,3\%), ensino fundamental $(40,8 \%)$ e renda entre $\mathrm{R} \$ 700,00$ e $\mathrm{R} \$ 1.200,00$ $(30,1 \%)$ (Tabela 1$)$.

Conforme demonstrado na Tabela 2, o número de gestações variou de um a sete, com prevalência de primípara $(27,2 \%)$ ou multípara $(28,2 \%)$ gestações. A maioria das mulheres $(57,3 \%)$ recebeu o diagnóstico de HIV antes do pré-natal. Embora 12,6\% tenham sido identificadas como usuárias de drogas injetáveis, grande parte dos prontuários $(72,8 \%)$ não possuía registro claro sobre esta variável. Setenta e sete porcento das mulheres $(77,7 \%)$ não apresentava histórico de aborto anterior. 
Observou-se que $45,6 \%$ das mulheres não tiveram outra IST, no entanto, $15,5 \%$ tiveram sífilis congênita. Cesárea não eletiva foi a forma de escolha para o nascimento $(53,4 \%)$, seguido de cesárea eletiva e parto normal, com 22,3 e $24,3 \%$, respectivamente.

Tabela 1: Perfil sociodemográfico e econômico das gestantes HIV positivo atendidas no Hospital Municipal, Niterói, RJ, 2009-2015.

\section{Variáveis}

n

Ano do Atendimento

2009

2010

2011

2012

2013

2014

2015

Procedência

Niterói

São Gonçalo

Maricá

Sem informação

Faixa Etária

18 a 20 anos

21 a 30 anos

31 a 40 anos

41 anos ou mais

Escolaridade

Analfabeta

Ensino fundamental

Ensino Médio

Nivel superior

Sem informação

Renda

$\mathrm{R} \$ 100,00$ a $\mathrm{R} \$ 600,00$

$\mathrm{R} \$ 700,00$ a $\mathrm{R} \$ 1.200,00$

$\mathrm{R} \$ 1.300,00$ a $\mathrm{R} \$ 1.800,00$

$\mathrm{R} \$ 1.900,00$ a $\mathrm{R} \$ 2.200,00$

$R \$ 2.300,00$ a $R \$ 2.800,00$

$\mathrm{R} \$ 2.900,00$ a $\mathrm{R} \$ 3.500,00$

Sem informação

\section{DISCUSSÃO}

Embora a notificação, das grávidas HIV positivo tenha sido introduzida no Brasil a partir do ano 2000, em Niterói, até 2014, observava-se ainda um processo lento de registros em decorrência a subnotificação. Não há dados recentes sobre
$\%$ a frequência de abandonos ou perdas de acompanhamento deste grupo de pacientes, sendo o último dado epidemiológico oficial fornecido pelo Ministério da Saúde datado de 2014. Em Niterói entre os anos de 2000 a 2012 foram notificados 5873 casos gestantes HIV positivo, sendo que a maior parte se encontrava na faixa etária de 20 a 29 anos perfazendo $52,9 \%$ do total de grávidas notificadas ${ }^{(2)}$.

No Estado do Rio de Janeiro, de 2000 até 2018 , foram identificados 10.936 casos de gestantes infectadas pelo HIV, sem distinção entre Municípios, e este número vem se mantendo estável nos últimos 10 anos. No ano de 2017 foram notificados 497 novos casos com uma taxa de TV detectada de 2,3 casos a cada 1000 nascidos vivos ${ }^{(11)}$.

Nossos resultados revelam um aumento na frequência absoluta de gestantes soropositivas atendidas nos anos de 2009 e de 2015. Entre os anos 2010 e 2014 observou-se uma queda drástica no atendimento. Essas variações ao longo dos anos demonstram a necessidade de intensificação da elaboração de estratégias sob o enfoque à prevenção e à redução do aumento crescente da TV.

Quando a origem geográfica das gestantes é verificada, o município de Niterói apresenta o maior número de gestantes HIV positivo, seguido dos municípios de Maricá e de São Gonçalo. Observa-se uma grande ausência destas informações nos prontuários dos pacientes, e também nos registros oficiais.

O perfil sociodemográfico referente à faixa etária mostrou a maior frequência de grávidas infectadas pelo HIV com idade entre 21 a 30 anos, o que consiste em um dado esperado, pois trata-se do ápice do período reprodutivo. O respectivo resultado aproxima-se dos dados encontrados na literatura, em uma pesquisa por Assis e colaboradores $^{(12)}$, sobre conhecimento e prática sexual de gestantes HIV positivo atendidas em um Hospital Universitário Federal localizado no Município do Rio de Janeiro, que observou uma faixa etária de 30 e 34 anos. Da mesma forma, pesquisadores ressaltaram em uma pesquisa no Hospital das Clínicas da Universidade de 
Tabela 2: Perfil clínico das gestantes HIV positivo, atendidas no Hospital Municipal, Niterói, RJ, 2009-2015.

$$
\text { Variáveis }
$$

n

Número de Gestações

Uma

Duas

Três

Quatro

Cinco

Sete

Momento do Diagnóstico de HIV em Relação ao Pré-Natal

Antes

Durante

No parto

Sem informação

Uso de Drogas Injetáveis

Não

$\operatorname{Sim}$

12,6

Sem informação

Abortos Anteriores

Não

Um

Dois

Três

Outra IST

Não

Sífilis

Herpes

Sem informação

Uso de Antibiótico

Não

Sim

Dado não encontrado

Tipo de Parto

Cesárea eletiva

Cesárea não eletiva
Parto normal

24,3

São Paulo (USP), identificando uma média de idade de 25 a 34 anos $^{(13)}$. Por outro lado, Menezes e colaboradores ${ }^{(14)}$, no estado do Pará, em um serviço de referência em saúde à mulher e da criança, foi avalia- do em sua maioria, jovens com idade entre 18 e 23 anos.

No Brasil, de uma forma geral, a faixa etária mais acometida pelo HIV/AIDS em mulheres está compreendida entre 20 a $24 \operatorname{anos}^{(11)}$. Tais observações mostram que a faixa etária das grávidas infectadas pelo HIV, atendidas no HMCT, segue a tendência nacional, com predomínio de mulheres jovens, demonstração a "juvenilização" da epidemia ${ }^{(15)}$. Com relação aos países latino-americanos, há discordância quanto à faixa etária. Em um estudo realizado no Chile, por Wu e colaboradores ${ }^{(16)}$, identificaram uma prevalência de gestantes infectadas entre 14 e 24 anos, quanto à Colômbia, segundo Gómez, Carrillo e Rodríguez $^{(17)}$ a maioria das mulheres estava na fase reprodutiva (15 a 49 anos), porém, o percentual de gestantes infectadas neste estudo foi baixo, alcançando um percentual de 1\%. Coincidentemente, na Argentina, uma pesquisa realizada mostrou uma redução na incidência de mulheres infectadas pelo HIV na idade reprodutiva ${ }^{(18)}$, e em Honduras, a média de idade foi de 26 anos e a faixa etária reprodutiva foi evidenciada com idades entre 19 a 38 anos, semelhantes aos nossos dados ${ }^{(19)}$.

Quanto à escolaridade, nossas investigações constataram que a predominância nos casos registrados ocorreu em grávidas HIV positivo com menos de oito anos de estudo, o que demonstra baixo nível educacional. O mesmo foi encontrado por Silva e colaboradores ${ }^{(20)}$, em um estudo epidemiológico desenvolvido no nordeste brasileiro, encontraram que $30,5 \%$ das gestantes infectadas pelo HIV tinham baixa escolaridade. Resultados semelhantes em Passos (MG), quando os autores Souza e colaboradores ${ }^{(21)}$ evidenciaram baixo nível escolar em $80 \%$ das grávidas investigadas. De igual maneira, no estudo em grávidas HIV positivo atendidas em uma maternidade pública de referência no Estado do Pará(22), foi observado que $50,4 \%$ de sua amostra total cursou o Ensino Fundamental e apenas 38,2\% o Ensino Médio. Segundo o MS ${ }^{(22)}$, que ao investigar a AIDS na população de gestantes no Brasil, observou que $30,1 \%$ das mulheres possuíam de 5 a $8^{a}$ série incompleta.

Desse modo, o grau de escolaridade vem sendo utilizado como um importante indicador comparativo as variáveis socioeconômicas. Dessa maneira, o aumento 
da proporção de casos de AIDS em indivíduos com menor grau de instrução tem sido um indicativo de extravasamento da epidemia para camadas desfavorecidas da sociedade, descritas como pobres ${ }^{(14)}$.

Quanto à renda, observou-se que a prevalência de gestantes HIV positivo declarou renda família, em torno de um salário mínimo. Foi identificado o mesmo achado, num estudo realizado em um centro de referência em saúde da mulher do município de Santarém ${ }^{(23)}$.

No presente estudo, observamos que a multigestação foi mais frequente que a gestação única, assim como não houve relatos de abortos anteriores, estes dados encontrados são diferentes, com relação a outro estudo de variáveis epidemiológicas, por Silva e colaboradores ${ }^{(20)}$, evidenciaram que $21,75 \%$ das gestantes eram primigestas e possuíram abortamentos em gestações anteriores.

Quando se analisa o momento do diagnóstico de HIV em relação ao pré-natal, pode-se constatar que o maior número de diagnósticos confirmatórios do HIV ocorreu antes do pré-natal. A cobertura de 57,3\% de identificação da infecção pelo HIV no pré-natal, neste grupo, mostra-se inferior ao encontrado no norte do Brasil, que foi de $75,1 \%^{(24)}$.

Todavia, nossos dados superaram as expectativas das pesquisas nacionais que, de acordo com as informações coletadas a partir do Estudo Sentinela de Parturientes infectadas pelo HIV realizado no Brasil, revelaram que a cobertura efetiva de sorologia para o HIV na gestação foi de $52 \%{ }^{(25)}$. Sabe-se que entre 30 a $40 \%$ das mulheres soropositivas brasileiras, já obtiveram conhecimento acerca da própria infecção (HIV) antes da gravidez ${ }^{(26)}$.

Infelizmente, ainda há taxa considerável de mulheres sem o diagnóstico durante o pré-natal e parto, como há registro as nossas investigações, provavelmente devido a não realização do exame ou relacionado à própria conduta da assistência pela equipe de saúde. Entre os possíveis fatores que contribuem para a não totalidade da cobertura de detecção do HIV durante a gestação, ressalta-se: a ausência de pré-na-
A existência

concomitante do

HIV com outras

infecções durante a

gravidez gera muitas

intercorrências

durante a gestação e

parto. No presente

estudo, observa-se

uma prevalência

para sífilis, dado

relevante, uma

vez que a sífilis

apresenta risco de

dano placentário e

aumento da TV do

HIV intrauterino,

e também risco da

sífilis congênita ${ }^{(30)}$. tal, a ausência do pedido do teste, a negação das gestantes e o desconhecimento do resultado no parto ${ }^{(25)}$.

Mesmo que, a disponibilidade do teste anti-HIV na assistência pré-natal da rede pública exista desde 1998, verificou-se que grande parte das mulheres desconhecem a possibilidade de realização do teste ${ }^{(27)}$. Esta é uma circunstância delicada, uma vez que demonstra a necessidade de alcançar um maior número de pessoas, por causa do risco de transmissão direta e repercussões importantes para o binômio mãe-filho, igualmente para a saúde pública, e indica uma lacuna na implementação desta prática na atenção básica ${ }^{(28)}$.

Nos nossos estudos, a maioria das gestantes não possuía registro claro de drogas injetáveis, embora uma pequena parte era usuária de drogas. Em contrapartida, o estudo por Barbosa e colaboradores ${ }^{(29)}$, envolvendo os fatores de risco relacionados à TV do HIV apontou que $10 \%$ delas relataram uso de drogas injetáveis durante o período gestacional, e esse é um importante fator na caracterização dos hábitos de saúde dessas mulheres. É relevante o número de usuárias de substâncias psicoativas nessa situação, e estas tendem a apresentar deficiência no autocuidado. $\mathrm{O}$ tratamento adequado diante as condutas de profilaxia da $\mathrm{TV}$, tem probabilidade de diminuir a cadeia de transmissão do HIV por meio do uso de drogas parenterais ou injetáveis.

A existência concomitante do HIV com outras infecções durante a gravidez gera muitas intercorrências durante a gestação e parto. No presente estudo, observa-se uma prevalência para sífilis, dado relevante, uma vez que a sífilis apresenta risco de dano placentário e aumento da TV do HIV intrauterino, e também risco da sífilis congênita ${ }^{(30)}$. Os dados apresentados ainda foram mais significativos do que um estudo transversal no serviço de referência em Ambulatório de Moléstias Infectocontagiosas em Ginecologia e Obstetrícia no Hospital das Clínicas da Faculdade de Medicina de Ribeirão Preto da Universidade de São Paulo (USP), no qual encontrou-se 5,3\% de sífilis diagnosticada ${ }^{(13)}$. 
Quanto ao tipo de parto realizado nestas mulheres, os resultados nos prontuários mostram que, no HMCT, 24,3\% foram submetidas ao parto vaginal, $22,3 \%$ à cesárea eletiva e 53,4\% à cesárea não eletiva. Burges e Weiser ${ }^{(31)}$ sugerem como melhor opção a cesariana eletiva antes do trabalho de parto, ou mesmo nos primeiros estágios do mesmo. A escolha da forma de nascimento tem influência na transmissão do HIV para a criança, devido ao tempo de contato com o sangue e fluidos maternos contaminados.

Em um estudo de metanálise, realizado por The International Perinatal HIV Group $^{(32)}$, foi destacado que os procedimentos que envolvem o parto possuem extrema relevância na TV. Inicialmente, os resultados de 15 estudos revelaram que a cesariana eletiva, realizada antes da rotura das membranas amnióticas, diminuiu o índice de transmissão do HIV em $50 \%$, quando comparado com as demais formas de partos. Além disso, propôs que a cesariana eletiva, juntamente ao uso de protocolo que associa a terapia TARV até o parto à dose intravenosa administrada antes e durante o parto, propiciou a redução da transmissão em até $90 \%{ }^{(32)}$. Em seguida, outra metanálise publicada pelo grupo Cochrane, em $2008^{(33)}$, analisou 26 estudos que certificaram a eficácia e a segurança da cesariana eletiva na prevenção da TV do HIV.

Todos os estudos corroboraram que a cesariana eletiva foi eficaz para a prevenção da TV do HIV nas mulheres que não fizeram uso de ARV na gestação, e naquelas que usaram apenas zidovudina (AZT).
Porém, esta mesma análise verificou-se uma maior probabilidade de morte para a mãe devido às complicações da cesariana eletiva, tais como sepse, endometrite e tromboembolismo ${ }^{(33)}$.

Chama-se atenção neste trabalho o fato de que a maior parte das grávidas não fez acompanhamento do programa IST/HIV. A quantidade elevada dessas mulheres que não participaram do programa é um fato preocupante, pois significa o não desenvolvimento das medidas profiláticas para redução TV do HIV/AIDS. Dessa forma, é possível questionar se as mesmas também foram orientadas quanto aos contraceptivos e à prevenção de ISTs, o que representaria uma violação nos direitos sexuais e reprodutivos e de saúde desses indivíduos ${ }^{(30)}$.

Com isso, observa-se que a verdadeira necessidade de informar a população sobre HIV/AIDS, no intuito de otimizar a adesão das mulheres ao pré-natal, bem como ao tratamento.

\section{CONCLUSÃO}

As gestantes HIV positivo acompanhadas no presente estudo estão estão incluídas na faixa etária 21 a 30 anos, predominando grávidas de procedência local, em situação socioeconômica de vulnerabilidade, com baixo nível de escolaridade, e o diagnóstico anterior precedem a gestação atual.

Em relação à forma de contágio, a utilização de drogas e de abusos não foi significativa. A contaminação foi mais evidente através das relações sexuais com parceiros únicos e múltiplos infectados.
$\mathrm{O}$ número de gestantes que realizou o pré-natal, e à terapêutica TARV na gestação e no parto foi alto, apesar de não terem participado do acompanhamento no programa de IST/AIDS. A frequência de doenças sexualmente transmissíveis concomitantes foi elevada.

A abrangência dos resultados deste estudo refere-se à fonte secundária das informações, além disso, o elevado número de subregistros em alguns itens compromete a qualidade da avaliação e reafirma o alcance de estudos documentais. Há nítida necessidade do melhoramento das informações adquiridas por esses bancos de dados assim como a capacitação dos profissionais de saúde que suprem as medidas essenciais para a avaliação mais adequada da prevenção da TV do HIV. Mostra-se relevante a realização de pesquisas epidemiológicas desse tipo, pois evidenciam a realidade local, viabilizando aos gestores e profissionais envolvidos, como os multiprofissionais, a avaliação das questões em análise e o planejamento e execução de medidas preventivas guiadas.

\section{AGRADECIMENTOS}

Os autores agradecem à enfermeira Lorrana da Silva Motta e ao Marcio Oliveira Ramos responsável pelo setor de Bioestatística do Hospital Municipal Carlos Tortelly, ao Erick da Silva Bernardes pela revisão do texto em português, e ao Programa de Mestrado Profissional em Saúde Materno Infantil (Faculdade de Medicina/UFF).

\section{REFERÊNCIAS}

1. Sabino, E. C.; Barreto, C. C.; Sanabani, S. S. Infecção por HIV e AIDS. In: Focaccia, R. Veronesi: Tratado de infectologia. 4 ed. São Paulo: Ediatora Atheneu, 2009.

2. Boletim Epidemiológico HIV/AIDS. Ministério da Saúde. Coordenação Nacional de DST e AIDS. Brasília (DF); 2014.

3. Duarte G. Síndrome da Imunodeficiência Adquirida. In: Monteleone PPR, Valente CA. Infectologia em Ginecologia e Obstetrícia. São Paulo: Atheneu, 1997. P. 198-218.
4. Goldmeier MK. Dificuldade de adesão ao tratamento antirretroviral sob opinião da enfermagem. Boletim da saúde. jul/ dez.2003; v.17,n.2.

5. Joint United Nations Programe on HIV/AIDS (UNAIDS). Data 2017. Program HIV/AIDS [Internet]. 2017;1-248. Available from: http://www.unaids.org/sites/default/files/media_asset/20170720_Data_book_2017_en.pdf

6. Sbalqueiro R, Reggiani C, Tristão E. Estudo da prevalência e variáveis epidemiológicas da infecção pelo HIV em gestantes aten- 


\section{REFERÊNCIAS}

didas na maternidade do Hospital de Clínicas de Curitiba. DST J Bras Doenças. 2004;16(2):40-7.

7. Calazans, G. et al. Plantões Jovens: acolhimento e cuidado por meio da educação entre pares para adolescentes e jovens nos Centros de Testagem e Aconselhamento - CTA. Saúde e Sociedade, v.15, n.1, p.22-36, jan/abr, 2006.

8. Barbosa Junior A, Szwarcwald CL, Pascom ARP, Souza Júnior PB. Tendências da epidemia de AIDS entre subgrupos sob maior risco no Brasil, 1980-2004. Cad Saúde Pública. 2009;25(4):72737.

9. Brito AM, Castilho EA, Szwarcwald CL. AIDS e infecção pelo HIV no Brasil: uma epidemia multifacetada. Rev Soc Bras Med Trop. 2000;34(2):207-17.

10. Fonseca MGP, Bastos FI. Twenty-five years of the AIDS epidemic in Brazil: principal epidemiological findings, 1980- 2005. Cad Saúde Pública. 2007; 23 (suppl. 3): S333-S343.

11. Boletim Epidemiológico - AIDS. Ministério da Saúde. Secretaria de Vigilância em Saúde - Departamento de Vigilância, Prevenção e Controle das Infecções Sexualmente Transmissiveis, do HIV/AIDS e da Hepatites Virais. Brasília (DF) 2018.

12. Assis MR De, Lima DS, Paiva MS. Conhecimento e prática sexual de gestantes HIV positivo atendidas em hospital universitário Resultados e Discussão Caracterização dos sujeitos. 2016;24(6):1-6. DOI: http://dx.doi org/10. 12957/re-Uerj.2016.12536.

13. Pimentaa ATM, Duarteb G, Couto-Fernandezc JC, Corread IA, Mellie PP dos S, Quintana SM. Hiv-Infected Pregnant Women Attending Reference Service: Clinical and Sociodemographic Features. Rev Atenção à Saúde. 2015;13(45):20-5. DOI: 10.13037/ rbcs. vol13n45.2719.

14. Boletim Epidemiológico HIV/AIDS. Ministério da Saúde. Coordenação Nacional de DST e AIDS. Brasília (DF); 2017.

15. Figueiró-Filho EA, Senefonte FRA, Lopes AHA, GS Júnior V, Botelho CA, Duarte G. Perfil epidemiológico da infecção pelo HIV1 em gestantes do estado de Mato Grosso do Sul - Brasil. DST - J Bras Doenças Tropicais. 2005; 17(4): 281-287.

16. Wu E, Galaz MI, Larranaga C, Chavez A, Gonzalez M, Alvarez AM, et al. Infeccion por VIH/SIDA en ninos y adolescentes: cohorte chilena 1987-2014. Rev Chilena Infectol. 2016; 33(Supl1):11-19.

17. Gómez-Ríos AC, Carrillo-Rozo ME, Rodríguez-Ortiz JA. Morbimortalidad materno-perinatal en pacientes con infección por VIH, Hospital Simón Bolívar, 2003-2011, Bogotá (Colombia). Rev Colomb Obstet Ginecol. 2014;65(4):308.

18. Antropológicos H, Alegre P. Cuerpo y narrativa. 15:247-72.

19. Parham L, Murillo W, Rivera IL. Resistencia del VIH en mujeres embarazadas de Honduras durante el ano 2015. Rev Cienc Tecnol. 2016.

20. Silva CM da, Alves R de S, Santos TS Dos, Bragagnollo GR, Tavares CM, Santos AAP Dos. Epidemiological overview of HIV/ AIDS in pregnant women from a state of northeastern Brazil. Rev
Bras Enferm. 2018;71(suppl 1):568-76.

21. Souza CP, Piantino CB, Queiroz CA, Maia MAC, Fortuna CM, Andrade RD. Incidência de transmissão vertical do HIV entre gestantes soropositivas cadastradas em um serviço de referência regional. J Res: Fundam Care. 2016;8(2):4526-37.

22. Menezes L do S, Palácios VR, Alcântara MS, Bichara CN. Prevalência da infecção Por Hiv em Grávidas no norte do Brasil. J Bras Doenças Sex Transm. 2012;24(4):250-4.

23. Nascimento VB, Nascimento NVM, Oliveira JSS, $114 \mathrm{~J}$ Health Sci Inst. 2018; 36(2): 109-14 Bezerra LO, Faria DN, Ciosak $\mathrm{SI}$, et Aspectos epidemiológico e clínico de gestantes com HIV/ AIDS atendidas em um centro de referência em saúde da muIher do município de Santarém, Pará, Brasil. Health Sci Inst. 2018;36(2):109-14.

24. Menezes LSH, Palacios RCM, Peixoto AS, Alcântara MSC, Bichara CNC. Perfil epidemiológico de grávidas HIV positivo atendidas em maternidade pública de referência. J.bras Doenças Sex Transm. 2012;24(4):250-254.

25. Souza Junior PRB, Szwarcward CL, Barbosa Júnior A, CarvaIho MF, Castilho EA. Infecção pelo HIV durante a gestação: estudo-sentinela parturiente, Brasil, 2002. Rev Saúde Pública Rio de Janeiro. 2004; 38(6): 764-72.

26. Silva NEK, Alvarenga AT, Ayres JRCM. Aids e gravidez: os sentidos do risco e o desafio do cuidado. Rev Saúde Pública. 2006;40(3):474-81.

27. Morimura MCR, Mendes MDC, Souza Al, Alencar LCA. Frequência de testagem rápida para o HIV durante a admissão para o parto em puérperas no Instituto Materno Infantil Prof. Fernando Figueira, IMIP. Rev Bras Matern infant. Recife. 2006; 6(Supl. 1):569-576.

28. Darmont MQR, Martins HS, Calvet GA, Deslandes SF, Menezes JA. Adesão ao pré-natal de mulheres HIV+ que não fizeram profilaxia da transmissão vertical: um estudo sócio-comportamental e de acesso ao sistema de saúde. Cad. Saúde Pública Rio de Janeiro. 2010; 26(9): 1788-1796.

29. Barbosa BLFA, Marques AK, Guimarães JV et al. Gestantes HIV Positivo e os Fatores de Risco Relacionados à Transmissão Vertical do HIV. Rev enferm UFPE on line., Recife, 12(1):171-8, jan., 2018.

30. DST/AIDS. BM da SS de V em SPN de. Recomendações para profilaxia da transmissão vertical do hiv e terapia antirretroviral em gestantes - 2010. Aids. 2010;1-146.

31. Burges H, Weiser B. Biology of HIV-1 in women and men. Clin Obstet Gynecol. 2001 jun; 44(2):137-43.

32. The International Perinatal HIV Group. The mode of delivery and the risk of vertical transmission of human immunodeficiency virus type 1: a meta-analysis of 15 prospective cohort studies. N Engl J Med. 1999 abr; 340(13): 977-87.

33. Read JS, Newell ML. Eficacia y seguridad del parto por cesárea para la prevención de la transmisión maternoinfantil del VIH-1 (Revisión Cochrane traducida). En: La Biblioteca Cochrane Plus, 2008; 4. 